\title{
Monitoramento de bacias hidrográficas para identificar fontes de sedimentos em suspensão
}

\author{
Catchment monitoring to trac suspended sediment sources
}

\author{
Jean Paolo Gomes Minella ${ }^{\text {* }}$ Gustavo Henrique Merten ${ }^{\mathrm{II}}$
}

\section{- REVISÃO BIBLIOGRÁFICA -}

\section{RESUMO}

Programas de conservação do solo e da água que utilizam a bacia hidrográfica como unidade de planejamento têm sido amplamente empregados. Um componente importante desses programas se refere à implantação de projetos de monitoramento hidrossedimentométrico e de qualidade de água para avaliar o impacto nos recursos hídricos das práticas introduzidas. Entretanto, em alguns casos, os resultados obtidos pelos projetos de monitoramento têm sido pouco conclusivos, devido a limitações dos procedimentos experimentais adotados. Esta revisão explora metodologias de avaliação que combinam técnicas tradicionais de monitoramento com técnicas de identificação de fontes de sedimentos que contribuem para elucidar os efeitos das práticas conservacionistas na produção de sedimentos em bacias hidrográficas e também a interrelação dinâmica entre as fontes de sedimentos.

Palavras-chave: conservação do solo, qualidade da água, produção de sedimentos.

\section{ABSTRACT}

Soil and water conservation programs frequently use catchments as planning units. An important follow-up component of these programs is the installment of hydrosedimentometric and water quality monitoring projects to evaluate the impact of the practices introduced. However, in some cases, these monitoring projects have yielded inconclusive results, mostly due to procedural limitations. This review explores methods that combine traditional monitoring techniques with sediment source identification to further elucidate the impact of conservation practices on sediment yield in the catchment and dynamic interactions between different sediment sources.

Key words: soil conservation, water quality, sediment yield.

\section{INTRODUÇÃO}

Atualmente, o planejamento do espaço rural e urbano necessita de ferramentas de análise capazes de avaliar quantitativamente os fatores que interferem na degradação dos solos e recursos hídricos de forma integrada. A erosão observada nas lavouras, nas estradas e nas áreas de construção está, evidentemente, relacionada com os impactos observados nos rios, tanto sob o aspecto físico, como químico e biológico. Entretanto, a conectividade entre os problemas que observamos nas vertentes e os problemas que encontramos nos rios não é direta. Mecanismos complexos de conectividade entre vertentes e rios controlam o comportamento hidrossedimentológico das "bacias hidrográficas". O estudo da variabilidade da produção de sedimentos tem contribuído para a compreensão dos fatores controladores dos processos erosivos e do transporte de sedimentos e poluentes nas bacias hidrográficas. A identificação das fontes de sedimentos é uma técnica que possibilita localizar e quantificar as fontes de sedimentos dentro de uma bacia, contribuindo para descrição dos processos de ligação entre calha e vertente e para o planejamento dos recursos naturais.

Neste trabalho de revisão bibliográfica, são apresentados os componentes e os fatores condicionantes envolvidos com a estimativa da produção de sedimentos, salientando os efeitos do

'Departamento de Solos, Universidade Federal de Santa Maria (UFSM). Av. Roraima, 1000, 97105-900, Santa Maria, RS, Brasil. Email: jminella@ smail.ufsm.br. *Autor para correspondência.

"Departamento de Obras Hidráulicas, Instituto de Pesquisas Hidráulicas, Universidade Federal do Rio Grande do Sul (UFRGS), Porto Alegre, RS, Brasil. 
manejo do solo na escala de bacia hidrográfica. $\mathrm{Na}$ sequência, são apresentados os aspectos que norteiam o planejamento do monitoramento para que a aquisição de dados corresponda aos objetivos requeridos. Nesta etapa, é apresentado um resumo das técnicas de identificação das fontes de sedimentos e, finalmente, são listadas as principais tendências na análise da produção de sedimentos na escala de bacia hidrográfica.

\section{DESENVOLVIMENTO}

Produção de sedimentos

O crescimento agrícola e urbano e a consequente ocupação das bacias hidrográficas, com a alteração do uso do solo, promovem grande impacto negativo ambiental e socioeconômico à humanidade, que é a erosão e a presença de sedimentos nos rios. Entretanto, as relações entre os fatores interferentes (clima, relevo, solo e uso) e a produção de sedimentos ainda representam um desafio para a pesquisa.

Define-se "Produção de Sedimentos" (PS) como a quantidade de sedimentos que é removida para fora de uma bacia hidrográfica ou de uma área considerada. A PS representa uma parcela do total do material que foi erodido na bacia vertente e no canal fluvial (erosão bruta) e transferido até uma determinada seção do canal fluvial. Em termos quantitativos, a PS é a diferença entre a erosão bruta e a quantidade de sedimentos depositada na bacia vertente de uma determinada seção do canal fluvial (WALLING, 1983).

Na prática, a PS é dificilmente medida ou estimada devido à complexidade dos processos de propagação e de deposição dos sedimentos erodidos na bacia hidrográfica. Alternativamente, utiliza-se um conceito empírico para estimar um valor adimensional, o qual representa a parcela do total da erosão bruta que chega ao exutório da bacia (VANONI, 1975, WALLING, 1983; 1990). Esse valor é conhecido como Taxa de Emissão de Sedimentos - TES (Sediment Delivery Ratio - SDR), que é igual a razão entre a produção de sedimentos no exutório (PS) e a erosão bruta na bacia (EB).

Componentes na estimativa da produção de sedimentos A produção de sedimentos é função dos processos erosivos que ocorrem nessa bacia, ou seja, das taxas de desagregação do solo por ação da precipitação e do escoamento laminar e concentrado, dos processos de transferência dos sedimentos da bacia vertente para a calha fluvial e pelos processos de erosão e deposição que ocorrem na calha fluvial.
Os processos relacionados com a desagregação do solo devido à ação do impacto das gotas da chuva e do escoamento superficial difuso e concentrado têm sido descritos com grande detalhamento (FOSTER, 1982; MORGAN, 2005). Da mesma forma, a mecânica fluvial tem descrito os processos relacionados com o transporte e deposição dos sedimentos que se encontram em trânsito na calha fluvial (KNIGHTON, 1998; SILVA et al., 2003). Por outro lado, os processos envolvidos na transferência dos sedimentos das áreas fontes para a calha fluvial são muito pouco conhecidos, mesmo que isso seja de fundamental importância para estimar a produção de sedimentos (WALLING, 1990).

Os resultados obtidos em parcelas experimentais de erosão providenciam uma clara evidência dos efeitos do uso e manejo dos solos sobre as taxas de erosão. Entretanto, quando alteramos a escala de análise, saindo das parcelas experimentais para a escala de bacia, as evidências do impacto do uso e do manejo do solo na produção de sedimentos são menos claras. A complexidade do relevo, os fragmentos de vegetação e as construções humanas (estradas, drenos, barreiras, etc.) afetam a conectividade entre as vertentes e a rede de drenagem. Nesse contexto, a conectividade é um conceito que representa a facilidade em que os sedimentos erodidos são transferidos das fontes para a rede de drenagem (WALLING, 1990, 1999).

Fatores interferentes da produção de sedimentos

Os fatores controladores da magnitude e da característica da produção de sedimentos são o clima, as características fisiográficas e o uso e manejo do solo (MORGAN, 2005). A influência do clima pode ser relacionada com a produção de sedimentos por meio das características da precipitação (volume, intensidade e frequência). As características da precipitação irão determinar, principalmente, a magnitude da PS. A fisiografia da bacia (declividade do terreno, atributos do solo, características da rede de drenagem, etc.) irá determinar a capacidade de transferência dos sedimentos pelas vertentes e rios. O efeito do uso e manejo dos solos sobre a PS tem a propriedade de atenuar ou agravar os fatores anteriores (VERSTRAETEN et al., 2006). A alteração da cobertura vegetal natural para o uso agrícola, por exemplo, pode modificar a PS em várias ordens de magnitude, em comparação com uma área não perturbada (MORGAN, 2005).

Qualquer preocupação em relatar as mudanças de produção de sedimentos em uma bacia deve considerar os processos que interferem na Taxa 
de Emissão de Sedimentos (SDR). Dependendo das características da bacia, parte dos sedimentos erodidos durante o período de erosão acelerada pode ficar depositado no fundo de vales e dentro do canal e não chegar ao exutório da bacia. Reciprocamente, a redução na perda de solo pela implementação de medidas conservacionistas e melhorias das práticas de cultivo podem não refletir na redução da produção de sedimentos, porque os sedimentos armazenados nos antigos depósitos podem ser remobilizados (TRIMBLE, 1983; HADLEY et al., 1985). Por isso, a dimensão temporal do efeito do uso e do manejo do solo sobre a produção de sedimentos também precisa ser considerada. Em muitas áreas, o impacto da atividade humana pode ser relativamente recente e em outras as mudanças no uso do solo podem estar ocorrendo durante séculos (YAN et al., 2010).

A literatura mostra claramente o forte efeito do uso do solo sobre a erosão hídrica, mas todos são unânimes em considerar que existem incertezas no estabelecimento do grau de influência desse controle sobre a variabilidade da PS.

São necessários estudos dos efeitos qualitativos do uso do solo na erosão hídrica (MERTEN \& MINELLA, 2005), cujo conhecimento deve ser utilizado para os seguintes propósitos: convencer dos gestores e dos agricultores acerca dos impactos locais e regionais da erosão; criar condições para implementação das práticas conservacionistas do solo; e demonstrar o custo-benefício dos projetos de redução da produção de sedimentos. A compreensão dos efeitos qualitativos do uso do soloé um mecanismo importante na estratégia para implementar práticas de conservação na escala de bacia, porque são os agricultores que definem a organização espacial e temporal de sua propriedade. Quando ocorre o planejamento correto de uma propriedade agrícola, utilizando técnicas adequadas de ocupação e manejo do solo, de acordo com sua aptidão agrícola, a PS pode se aproximar aos valores de áreas preservadas (MORGAN, 2005).

A partir de uma perspectiva que considera também aspectos qualitativos do uso, manejo e ocupação do solo, é preciso desenvolver metodologias de monitoramento e modelagem da PS que incluam as características e processos internos da bacia hidrográfica, especialmente dos processos referentes à transferência de sedimentos da bacia vertente para a calha fluvial, além dos processos monitorados no exutório (MINELLA et al., 2008). Segundo WALLING (1990), a eficiência nas estratégias de controle dos sedimentos erodidos depende do aperfeiçoamento da obtenção de informações dos vários processos que afetam a emissão de sedimentos por uma bacia, incluindo a identificação da contribuição das fontes primárias de sedimentos.

Os estudos na escala de bacia vertente contribuem para entender os processos de erosão e mobilização de sedimentos, porque considera a complexidade natural dessa escala, a variabilidade climática natural e o efeito das atividades antrópicas na vertente ao longo do tempo. Entretanto, os processos que ocorrem na escala de bacia hidrográfica (bacia vertente mais canal fluvial) são ainda mais complexos, porque incluem a interação entre as duas escalas.

Monitoramento hidrossedimentométrico

A capacidade de compreensão de como os processos hidrossedimentológicos de uma bacia hidrográfica respondem aos efeitos do uso e manejo do solo depende, fundamentalmente, do monitoramento adequado de variáveis que estão envolvidas no processo. No caso dos processos hidrossedimentológicos, as principais variáveis são a precipitação, a vazão, a concentração de sedimentos em suspensão e a descarga de sedimentos de arraste (VANONI, 1975). Por razões logísticas e financeiras, as medições se concentram no exutório da bacia, onde os processos e distúrbios que ocorrem na bacia vertente são integrados com aqueles que ocorrem na calha fluvial e expressos em termos de alterações da vazão e da concentração de sedimentos.

A metodologia tradicional de monitoramento hidrossedimentométrico é baseada na obtenção de uma série temporal de dados de precipitação, vazão e concentração de sedimentos que possibilita o cálculo do fluxo de sedimentos. Esse fluxo é caracterizado pela descarga sólida em suspensão (massa por unidade de tempo) e a produção de sedimentos, que é a integração do fluxo no tempo (VANONI, 1975). A qualidade dos resultados será melhor quanto maior for a frequência de amostragem. Um número maior de amostragem em um menor intervalo de tempo aumenta a probabilidade de coincidir o momento da coleta com os eventos de cheia, os quais são responsáveis pela maior parte da produção de sedimentos. WALLING (1990), por exemplo, salienta que a frequência de amostragem afeta sensivelmente a estimativa do fluxo em suspensão. Uma estimativa baseada em amostragem trimestral não corresponde à estimativa de amostragem semanal, diária ou horária, podendo haver uma subestimativa da produção de sedimentos quanto maior for o intervalo entre as medidas (HOROWITZ, 2003; MERTEN et al., 2006). Em um programa de monitoramento hidrossedimentométrico de alta frequência, as principais limitações estão relacionadas com o 
deslocamento até a seção de monitoramento e ao alto custo de coleta e da análise da concentração de sedimentos em suspensão.

Como alternativa ao monitoramento manual, existe a possibilidade de agregar equipamentos automáticos que complementarão a obtenção de dados, permitindo coletar dados na ausência do hidrotécnico. Uma destas tecnologias é a turbidimetria, que tem sido considerada como uma técnica promissora na estimativa da concentração de sedimentos em suspensão. $\mathrm{O}$ equipamento mede a propriedade ótica que causa a dispersão ou absorção de luz pelas partículas em suspensão, sendo simples, barato de operar e permite a coleta contínua de dados (GLYSSON \& GRAY, 2002; MINELLA et al., 2007). O valor da turbidez é, então, correlacionado com a concentração de sedimentos obtidos em alguns eventos de cheia. É importante salientar que a utilização de turbidímetros é uma metodologia complementar para o monitoramento da concentração de sedimentos em suspensão, devendo ser realizado em conjunto com técnicas tradicionais de coleta de dados, para a verificação e calibração dos turbidímetros.

Identificação das fontes primárias dos sedimentos em suspensão

As técnicas de identificação de fontes têm contribuído para esclarecer como os diferentes sistemas que compõem a bacia hidrográfica contribuem para a produção de sedimentos na rede de drenagem. Segundo a definição de COLLINS (1997), a técnica de identificação de fontes (fingerprinting approach) usa a dimensão qualitativa dos sedimentos para elucidar a sua origem pelo exame detalhado de várias propriedades numa determinada escala espacial e temporal. Inúmeros trabalhos têm demonstrado que a utilização de técnicas de traçamento de sedimentos contribui para quantificar a contribuição das suas diferentes fontes para a rede de drenagem (COLLINS et al., 1997; WALLING, 2005). Essa informação tem grande relevância, pois possibilita compreender a dinâmica do processo hidrossedimentológico, como o mecanismo de conexão entre as vertentes e a calha fluvial na rede de drenagem (WALLING, 1999). Resumidamente, as vantagens da identificação de fontes de sedimentos são: (a) identificação dos locais geradores de sedimentos na bacia e da quantidade deles erodido; b) contribuição para o desenvolvimento de modelos com discretização espacial da produção de sedimentos, os quais são valiosos para interpretação dos dados de sua produção e previsão; (c) contribuição para o entendimento da dinâmica dos sedimentos em bacias; (d) desenvolvimento de programas de manejo integrado de bacias hidrográficas; (e) delineamento de estratégias efetivas para o controle da erosão e poluição difusa.

A evolução do método de identificação de fontes de sedimentos

Os estudos de identificação de fontes de sedimentos iniciaram com o desenvolvimento de métodos de traçamento do movimento de sedimentos em bacias a partir da década de 70. WOLMAN (1977) utilizou inicialmente a técnica com propriedades eletromagnéticas naturais presentes nos sedimentos. O fundamento teórico foi a investigação detalhada de propriedades naturais encontradas nos solos das fontes que poderiam ser comparadas com as variações das mesmas propriedades dos sedimentos transportados em suspensão nos rios.

Os estudos iniciais de identificação dessas fontes apresentaram resultados essencialmente qualitativos, mostrando quais delas seriam as mais importantes e, também, na descrição de mudanças que podem ocorrer dessas fontes durante os eventos ou entre estações do ano. A utilização de um número maior e heterogêneo de propriedades traçadoras e a incorporação de métodos quantitativos marcaram o segundo importante avanço metodológico dessa técnica, que ocorreu a partir do final da década de 80.

A procura por um número maior de propriedades traçadoras, as quais deveriam claramente distinguir as fontes potenciais de sedimentos, geraram um grande número de pesquisas que avaliaram um largo número de características presentes nos sedimentos e solos (WALLIN \& COLLINS, 2000). Essas características correspondem a diferentes grupos de parâmetros, os quais incluem parâmetros mineralógicos, magnéticos, geoquímicos, orgânicos, radiômetros, isótopos e físicos.

Os radionucleotídeos são considerados traçadores em potencial devido à sua distribuição homogênea sobre grandes áreas e seu comportamento estável no ambiente. Esses estudos utilizam principalmente o Césio-137, o Chumbo-210, o Berílio07 e o Rádio-226. Os radionucleotídeos são, provavelmente, a mais eficiente propriedade traçadora para a distinção entre fontes superficiais e subsuperficiais em bacias (ZAPATA, 2002). O uso de traçadores geoquímicos é baseado na exploração da composição química dos sedimentos (metais pesados, alcalinos, elemento traços, etc.) nos seus compartimentos geoquímicos (concentração associada aos óxidos, estrutura cristalina, total, etc.) (HOROWITZ, 1991). Os traçadores geoquímicos apresentam as vantagens de serem facilmente 
determinados e de sua variabilidade na bacia estar associada com a variabilidade do tipo de fonte (WALLING \& WOODWARD, 1995; COLLINS et al., 1997).

Em relação ao desenvolvimento dos métodos quantitativos, podemos citar a contribuição de YU \& OLDFIELD (1989). O trabalho desenvolveu um modelo estatístico multivariado para a análise de propriedades dos sedimentos erodidos em seis diferentes fontes. Esse trabalho elucidou o fato de que cálculos quantitativos são mais úteis do que descrições puramente qualitativas, permitindo identificar a proporção das fontes que estão contribuindo para os sedimentos presentes nos rios. Além desse, outros trabalhos contribuíram para o estabelecimento e refinamento do modelo multivariado de classificação, como, por exemplo, os discutidos por WALLING \& WOODWARD (1995).

O aumento no potencial de cálculo, disponibilizado pelos modelos multivariados de classificação, gerou a necessidade de procedimentos estatísticos mais rigorosos para selecionar as propriedades traçadoras a serem inseridas no modelo.

WALLING \& WOODWARD (1995)

testaram a eficácia do método de identificação de fontes de sedimentos para uma bacia de $276 \mathrm{~km}^{2}$ no Reino Unido. No estudo, foi utilizado um conjunto de nove propriedades traçadoras (três radionucleotídeos, quatro propriedades magnéticas dos minerais e dois elementos orgânicos). Sete tipos de fontes foram analisados, os quais representam áreas de lavoura e pastagem provenientes de três geologias e material proveniente de canais. A contribuição das fontes foi determinada em amostras de sedimentos em suspensão, coletadas durante eventos de cheia. Os resultados demonstram que foi possível documentar as alterações na contribuição relativa das fontes em resposta à origem do escoamento e ao tempo de percurso dos sedimentos de cada fonte. Considerando a geologia como tipo de fonte e a proporção do uso do solo em cada geologia, as contribuições das fontes foram: Cretáceo de 19,8\% (pastagem 9,2\% e áreas cultivadas 10,6\%), Triássico 41,8\% (pastagem 12,3\% e áreas cultivadas 29,5\%), Permiano 26,4\% (pastagem 6,7\% e áreas cultivadas $19,7 \%$ ) e dos canais de drenagem 12,0\%. É importante ressaltar que a obtenção de traçadores é facilitada quando existem fatores naturais ou antrópicos que determinam diferenças na composição química dos sedimentos, como, por exemplo, em grandes bacias que apresentam diferentes materiais geológicos. Em pequenas bacias, a obtenção de diferenças nos traçadores em geral é obtida em função do manejo do solo, da profundidade do fenômeno erosivo, umidade do terreno, os quais determinam diferenças nas características químicas dos solos e consequentemente dos sedimentos erodidos.

COLLINS et al. (1997) exploraram a importância do conceito de "identificação multiparamétrica", que preconiza a utilização de inúmeras características dos sedimentos como propriedades traçadoras em potencial. Nesse caso, são utilizados conjuntos distintos de propriedades traçadoras, por exemplo, elementos do grupo dos parâmetros geoquímicos, radionucleotídeos e características eletromagnéticas dos sedimentos. A consequência de um número maior de variáveis foi a diminuição das incertezas relacionadas com o processo de enriquecimento e o aumento da capacidade de discriminação entre as fontes. Apesar das vantagens, é necessário um procedimento estatístico mais apurado, devido a um número maior de variáveis. Um grande número de variáveis permite uma melhor discriminação das fontes e classificação das amostras de sedimentos em suspensão, entretanto não é desejável em função dos custos de análise, já que, em geral, as determinações das concentrações químicas de inúmeras amostras para um grande número de elementos pode inviabilizar o uso dessa metodologia. Sendo assim, é importante que o método estatístico determine o menor número possível de variáveis químicas que maximize a capacidade discriminante do conjunto de elementos. Outra contribuição de COLLINS et al. (1997) foi a análise da variabilidade temporal da contribuição das fontes inter e intra-evento. Os resultados demonstraram que a alteração da contribuição das fontes foi grande, tanto na escala intra-evento como na escala interevento. Os autores sugerem que a variabilidade interevento pode ser utilizada para descrever consequências importantes, relacionadas como o uso do solo e com o clima da região.

MINELLA et al. (2008) analisaram a origem dos sedimentos em suspensão de cinquenta eventos de precipitação que ocorreram entre maio de 2002 a março 2006 em uma bacia rural no noroeste do Rio Grande do Sul, numa condição de alta fragilidade ambiental. Tratava-se de produção de fumo em associações de Neossolos, Cambissolos e Argissolos e onde ocorreu uma mudança gradual de manejo de solo através da implementação de práticas conservacionistas (cultivo mínimo, pastagens de inverno para formação de palhada no período de cultivo, cultivo em nível). Avaliações hidrossedimentométricas foram realizadas através de medições de precipitação, volume escoado superficialmente, vazão máxima e produção de sedimentos dos eventos. Os resultados indicaram que 
tanto o volume de escoamento superficial como a vazão máxima diminui significativamente depois da implementação das práticas conservacionistas. Já em relação à produção de sedimentos nos eventos, verificou-se uma redução de aproximadamente $80 \%$ para os eventos de baixa magnitude e cerca de $40 \%$ para os eventos de média magnitude. Com relação à aplicação da técnica de identificação das fontes de sedimentos, verificou-se, para os períodos Pré- e Pósimplementação das práticas conservacionistas, uma diminuição da contribuição das lavouras e estradas de $62 \%$ e $36 \%$ para $54 \%$ e $24 \%$, respectivamente, enquanto a contribuição dos canais de drenagem aumentou de $2 \%$ para $22 \%$. O trabalho demonstra as vantagens do acoplamento das técnicas de identificação de fontes de sedimentos com técnicas tradicionais de monitoramento hidrossedimentométrico para elucidar não apenas os efeitos das práticas conservacionistas na produção de sedimentos da bacia, mas também a inter-relação dinâmica entre as fontes.

Síntese do método de identificação de fontes de sedimentos

A metodologia para identificar as fontes de sedimentos pode ser resumida por seis etapas principais (Figura 1). A primeira etapa consiste na determinação do problema erosivo na bacia com a seleção das fontes em potencial, que estão contribuindo com sedimentos para a rede de drenagem. Nessa etapa, é determinado o número de fontes que se deve incluir no estudo. As fontes de sedimentos em potencial são identificadas pela observação da mobilização e processos de transporte de sedimentos ocorrendo na bacia durante os eventos de chuva. Em geral, as bacias apresentam fontes de sedimentos além das lavouras, as estradas, rede de drenagem, áreas urbanas, etc.

$\mathrm{Na}$ segunda etapa, é estabelecida uma estratégia de amostragem dos solos nas diferentes fontes e de amostragem dos sedimentos em suspensão, utilizando o pressuposto de que os sedimentos em suspensão são compostos de uma mistura de sedimentos provenientes das fontes em potencial, previamente selecionadas. Para caracterizar os solos de cada fonte, é necessária uma malha de amostragem para representar toda a variabilidade espacial das fontes de sedimentos. O número de amostras dependerá da complexidade e da área da bacia. Quanto maior a heterogeneidade dos fatores fisiográficos, maior o número de amostras necessárias. Cada amostra deve ser composta por um conjunto de 10 subamostras, coletadas num raio de alguns metros. Para todas as análises (físicas e químicas), é necessário aproximadamente $0,5 \mathrm{~kg}$ de amostra coletada na superfície do solo $(0-5 \mathrm{~cm})$.

Na terceira etapa, são realizadas as análises químicas dos solos das fontes (HOROWITZ, 1991; WALLING \& COLLINS, 2000). Nessa etapa, é determinada a concentração de diversos elementos químicos, no intuito de encontrar aqueles que tenham a capacidade de distinguir as diferentes fontes. Para isso, é necessário considerar a estabilidade do elemento no ambiente, a qual é uma premissa fundamental dos traçadores. As amostras de solo devem ser peneiradas em malha de $2 \mathrm{~mm}$ para a determinação da textura e concentração química dos traçadores. Recomenda-se que a distribuição granulométrica deva ser realizada por granulômetro automático, em função da maior precisão e pelo maior número de classes. Como os elementos traços estão associados em maior concentração às partículas de tamanho silte e argila (HOROWITZ, 1991), existe a necessidade da aplicação de um fator de correção baseado na porcentagem de material menor que $0,063 \mathrm{~mm}$ para as amostras de soloe amostras de sedimentos em suspensão. A escolha dos elementos químicos deve ser baseada num conjunto de fatores que considera as possíveis diferenças nas

\begin{tabular}{|c|c|c|}
\hline $\begin{array}{l}\text { Seleção das fontes } \\
\text { Quais fontes em } \\
\text { potencial estáo } \\
\text { contribuindo com } \\
\text { sedimentos para a rede } \\
\text { de drenagem? }\end{array}$ & $\begin{array}{l}\text { Amostragem } \\
\text { Estratégia de amostragem } \\
\text { das fontes e dos } \\
\text { sedimentos em suspensão } \\
\text { para a sua caracterização } \\
\text { química e fisica }\end{array}$ & $\begin{array}{l}\frac{\text { Caracterização química e }}{\text { fisica dos solos das fontes }} \\
\text { Análises de laboratório } \\
\text { para a determinação da } \\
\text { concentração dos } \\
\text { traçadores. }\end{array}$ \\
\hline $\begin{array}{l}\text { Análise dos resultados } \\
\text { Contribuição absoluta } \\
\text { da produção de } \\
\text { sedimentos de cada uma } \\
\text { das fontes para o } \\
\text { periodo monitorado. }\end{array}$ & $\begin{array}{l}\text { Contribuição das fontes } \\
\text { Estabelecimento de uma } \\
\text { relação matemática entre } \\
\text { as concentraçós das } \\
\text { fontes e nos sedimentos } \\
\text { em suspensão. }\end{array}$ & $\begin{array}{l}\text { quimicos apresentam } \\
\text { propriedades traçadoras? } \\
\text { Análise estatistica para } \\
\text { definir o melhor conjunto } \\
\text { de propriedades } \\
\text { traçadoras. }\end{array}$ \\
\hline
\end{tabular}

Ciência Rural, v.41, n.3, mar, 2011. 
fontes de sedimentos. Selecionam-se elementos que apresentem diferenças de concentração entre as fontes, mas considerando os princípios de conservatividade. Os principais aspectos a serem considerados são: (a) infraestrutura disponível para análise e o custo de análise; (b) concentrações diferentes de elementos condicionados aos efeitos de calagem, adubação e aplicação de agroquímicos; (c) composição dos materiais das estradas, que é característica dos horizontes ou camadas mais profundos, os quais apresentam diferenças químicas que podem ser utilizadas para separar das outras fontes; (d) inclusão de elementos de grande conservatividade ambiental, que não estejam sujeitos à variação de sua concentração química, mesmo que permaneçam longo período em trânsito, desde sua origem até o exutório da bacia.

Na quarta etapa, é realizada a análise estatística dos dados para a escolha dos elementos traçadores. Essa etapa avalia a determinação da capacidade individual do elemento e posteriormente da capacidade do conjunto mínimo de elementos que maximiza a discriminação das fontes. A descrição matemática detalhada pode ser encontrada nos trabalhos de COLLINS et al. (1997), COLLINS \& WALLING (2002) e MINELLA et al. (2008). Para isso, podem ser utilizados dois testes estatísticos: i) o teste não paramétrico Kruskal Wallis e ii) uma análise de discriminação multivariada. O teste Kruskall-Wallis testa a hipótese nula em que as fontes são provenientes de uma mesma população (Equação 1). Para cada variável química determinada nos solos das fontes, o teste verifica sua capacidade em discriminar as fontes. Quando o valor de $\mathrm{H}$ calculado exceder o valor crítico, a hipótese nula é rejeitada e a variável apresenta capacidade discriminante entre as fontes.

$H=\frac{12}{n(n+1)} \sum_{s=1}^{k} \frac{R_{s}^{2}}{n_{l}}-3(n+1)$

em que, $R_{s}$ refere-se à soma dos ranks ocupados pela fonte $s$, sendo que $n_{l}$ é o número de observações da fonte $s, n$ a soma dos $n_{l}$ e $k$ o número de fontes.

Após ter avaliado individualmente os elementos com capacidade discriminante, ao conjunto selecionado de variáveis é imposto um teste estatístico multivariado para determinar o número mínimo de variáveis que maximiza a discriminação das fontes. $O$ parâmetro que controla a inclusão ou a exclusão de cada variável é a minimização do valor Wilks Lambda (JOHNSON \& WICHERN, 1992) (Equação 2).

$$
\Lambda^{*}=\frac{|W|}{|B+W|}
$$

em que $|\mathrm{W}|$ é o determinante da matriz da soma dos quadrados devido ao erro, e $|\mathrm{B}+\mathrm{W}|$ é o determinante da matriz da soma dos quadrados totais.

A quinta etapa é a determinação da proporção de cada fonte na composição dos sedimentos em suspensão. O modelo teórico que define a relação entre as concentrações dos traçadores nas fontes e nos sedimentos em suspensão é apresentado na equação 3. Algumas aplicações e variantes desse método estão descritos em WALLING \& WOODWARD (1995), COLLINS et al. (1997) e MINELLAet al. (2008) EWALLING \& COLLINS (2000).

$y_{i}=\sum_{s=1}^{n} a_{i s} P_{s} \quad(\mathrm{~s}=1,2, \ldots, \mathrm{n}) \mathrm{e}(\mathrm{i}=1,2, \ldots, \mathrm{m})$

em que: $y_{i}$ é o valor da variável i obtida no sedimento em suspensão, $a_{i s}$ são os coeficientes do modelo linear (concentração do elemento químico $i$ na fonte $s$ ), Ps é a proporção da massa da fonte $s$. A equação 3 pode ser apresentada como um conjunto de funções lineares de $m$ variáveis e $n$ fontes.

A solução do modelo acima é obtida por um processo iterativo de minimização de uma função objetiva que encontra a melhor proporção de cada fonte para compor uma concentração química semelhante à observada no sedimento em suspensão (Equação 4).

$$
f_{\min }=\sum_{i=1}^{m}\left\{\left(y_{i}-\left(\sum_{s=1}^{n} P_{s} a_{s i} z_{s}\right)\right) / y_{i}\right\}^{2}
$$

em que $y_{i}$ é a concentração do elemento $i$ na amostra de sedimento em suspensão; $P_{s}$ é a proporção de contribuição da fonte $s$ (variável desconhecida que o modelo estima por iteração); $a_{s i}$ é a concentração do elemento $i$ na fonte $s$; $z_{s}$ é um fator de correção da concentração química que considera a influência das diferenças na granulometria e/ou quantidade de matéria orgânica entre fonte e sedimento.

Esse é um processo matemático iterativo que cessa quando as diferenças entre as concentrações químicas dos sedimentos em suspensão calculadas pelo modelo e medidas na calha são minimizadas. O resultado da minimização da Equação 4 é a obtenção das porcentagens de contribuição relativa de cada fonte $\left(P_{s}\right)$. As equações podem ser compiladas em ambientes de programação. A descrição detalhada desta etapa é encontrada nos trabalhos de WALLING \& WOODWARD (1995), COLLINS et al. (1997) e MINELLA et al. (2008).

Tendo obtido as informações da contribuição de cada fonte para a produção de sedimentos na rede de drenagem, é possível melhorar a interpretação dos processos hidrossedimentológicos na bacia, os fatores condicionantes para a erosão, a 
mobilização e o transporte dos sedimentos para a rede de drenagem e, consequentemente, a elaboração de estratégias para controle das fontes de sedimentos.

\section{CONCLUSÃO}

A produção de sedimentos é reconhecida como uma informação importante para a gestão adequada dos recursos hídricos, entretanto existem poucas informações no Brasil sobre os aspectos relacionados com o uso e manejo do solo e a produção de sedimentos. Dificuldades relacionadas com métodos capazes de mensurar esses efeitos tanto no Brasil como em outros países estão entre os principais fatores que explicam esse problema. Para superar essa limitação, é necessário o desenvolvimento de métodos adequados para a realização de diagnósticos de bacia e com isso possibilitar um planejamento mais eficiente das medidas de controle da erosão e da produção de sedimentos.

As vantagens da técnica de identificação de sedimentos são as seguintes: (a) interpretar a dinâmica das fontes e processos de emissão; (b) elucidar a proveniência dos sedimentos nas escalas inter e intra-evento; (c) evitar problemas de falta de representatividade associada com as técnicas de modelagem e monitoramento tradicional; e (d) fornecer os detalhes requeridos para as necessidades atuais da pesquisa sobre o processo de emissão de sedimentos em bacias.

Entretanto, as técnicas de identificação de fontes de sedimentos apresentam um grau de complicação por um número potencial de problemas, como: (a) o processo seletivo da erosão hídrica (enriquecimento físico), aumenta os teores químicos dos sedimentos erodidos vinculados com o enriquecimento das partículas finas, impossibilitando a comparação direta entre sedimentos em suspensão e fontes; (b) a transformação das propriedades dos sedimentos pelo sistema fluvial; (c) o armazenamento e a subsequente remobilização pelo transporte fluvial.

A evolução de pesquisas para estabelecer informações de identificação das principais fontes de sedimentos combinado com o monitoramento hidrossedimentométrico podem contribuir para o desenvolvimento de estratégias mais eficientes para a melhoria do manejo dos solos nas bacias hidrográficas com implicações positivas aos recursos hídricos.

\section{REFERÊNCIAS}

COLLINS, A.L. et al. Source type ascription for fluvial suspended sediment based on a quantitative composite fingerprinting technique. Catena, Amsterdam, v.29, p.1-27, 1997. Disponível em: <http://www.linkinghub.elsevier.com/
retrieve/pii/S0169555X0600496X>. Acesso em: 15 abr. 2006. doi: 10.1016/j.geomorph.2006.10.018.

COLLINS, A.L.; WALLING, D.E. Selecting fingerprint properties for discriminating potential suspended sediment sources in river basins. Journal of Hydrology, Amsterdam, v.261, p.218-244, 2002. Disponível em: <http://www.linkinghub.elsevier.com/ retrieve/pii/S0022169402000112> Acesso em: 01 jul. 2008. doi: 10.1016/S0022-1694(02)00011-2.

FOSTER, G.R. Modeling the erosion process. In: HAAN, C.T. et al. (Ed.). Hydrologic modeling of small watersheds. Saint Joseph: ASAE, 1982. p.295-380.

GLYSSON, G.D.; GRAY, J.R. Total suspended solids data for use in sediment studies. In: Proceedings of the Federal Interagency Workshop on turbidity and other sediment surrogates. Reno, NV, 2002. Appendix 2.

HADLEY, R.F. et al. Recent developments in erosion and sediment yields studies. Paris: UNESCO, 1985. 127p. (Technical Documents in Hydrology).

HOROWITZ, A.J. A primer on sediment trace element chemistry. 2.ed. Chelsea: Lewis, 1991. 136p.

HOROWITZ, A.J. An evaluation of sediment rating curve for estimating suspended sediment concentration for subsequent flux calculation. Hydrological Process, v.17, p.3387-3409, 2003. Disponível em: www.water.usgs.gov/osw/techniques/TSS/ Horowitz.pdf. Acesso em: 30 mar. 2008. doi: 10.1002/ hyp. 1299.

KNIGHTON, D. Fluvial forms and processes: a new perspective. London: Arnold, 1998. 383p.

MERTEN, G.H.; MINELLA, J.P.G. Impact on sediment yield due to intensification of tobacco production in a catchment in southern Brazil. In: WALLING, D.E.; HOROWITZ, A.J. (Ed.). Sediment budgets. Wallingford: IAHS, 2005. V.2, n.292, p.239-244.

MERTEN, G.H. et al. Considerações sobre a utilizacao da curvachave de sedimentos para determinacao de fluxo de sedimentos. In: MERTEN, G.H. et al. (Ed). Sedimentos: o desafio da multidisciplinaridade. Porto Alegre: ABRH, 2006. p.8394.

MINELLA, J.P.G. et al. Combining sediment source tracing techniques with traditional monitoring to assess the impact of improved land management on catchment sediment yields. Journal of Hydrology, Amsterdam, v.348, p.546-563, 2008. Disponível em: <www.ufsm.br/fisicadosolo/ downloads/ Producao_Artigos/103.pdf>. Acesso em: 29 nov. 2009. doi: 10.1016/ j.jhydrol.2007.10.026.

MORGAN, R.P.C. Soil erosion and conservation. 3.ed. Malden: Blackwell, 2005. 304p.

OWENS, P.N.; COLLINS, A.J. Soil erosion and sediment redistribution in river catchments: measurement, modeling and management. Wallingford: CAB International, 2006. 328p.

SILVA, R.C.V. et al. Hidráulica fluvial. Rio de Janeiro: COPPE / UFRJ, 2003. V.1, 428p. 
TRIMBLE, S.W. A sediment budget for Coon Creek basin in the Driftless Area, Wisconsin, 1853-1977. American Journal of Science, New Haven, v.283, p.454-464, 1983.

VANONI, V.A. Sedimentation engineering. New York: ASCE, 1975. 745p. (Manuals \& Reports on Engineering Practice, n.54).

VERSTRAETEN, G. et al. The use of riparian vegetated filter strips to reduce river sediment loads: an overestimated control measure? Hydrological Processes, Chichester, v.20, p.42594267, 2006. Disponível em: <http://www.onlinelibrary.wiley.com/ doi/10.1002/hyp.6155/pdf>. Acesso em: 14 mar. 2009. doi: 10.1002/hyp.6155.

WALLING, D.E. The sediment delivery problem. Journal of Hydrology, Amsterdam, v.65, p.209-237, 1983. Disponível em: http://www.wou.edu/las/physci/taylor/g473/refs/ walling_1983.pdf. Acesso em: 18 nov. 2007. doi: 0022-1694/ 83

WALLING, D.E. Linking the field to the river: sediment delivery from agricultural land. In: WALLING, D.E. Linking land use, erosion and sediment yields in river basins. Hydrobiologia, Dordrecht, v.410, p.223-240, 1999.

WALLING, D.E. Tracing suspended sediment sources in catchments and river systems. Science of the Total Environment, Amsterdam, v.344, p.159-184, 2005. Disponível em: <http://www.linkinghub.elsevier.com/retrieve/ pii/S0048969705001063>. Acesso em: 05 abr. 2007. doi: 10.1016/j.scitotenv.2005.02.011
WALLING, D.E.; WOODWARD, J.C. Tracing sources of suspended sediment in river basins: a case study of the River Culm, Devon, UK. Marine and Freshwater Research, East Melbourne, v.46, p.327-336, 1995. Disponível em: <www.publish.csiro.au > Journals > Marine \& Freshwater Research>. Acesso em: 13 dez. 2007. doi:10.1071/MF9950327.

WALLING, D.E.; COLLINS, A.L. Integrated assessment of catchment sediment budgets: a technical manual. Exeter: University of Exeter, 2000. 168p.

WOLMAN, M.G. Changing needs and opportunities in the sediment field. Water Resources Research, Washington, v.13, p.50-54, 1977. Disponível em: <http://www.agu.org/ journals/ABS/1977/WR013i001p00050.shtml>. Acesso em: 25 abr. 2006. doi: 10.1029/ WR013i001p00050.

YAN, B. et al. Historical channel movement and sediment accreation along the South Fork of the Iowa River. Journal of Soil and Water Conservation, Ankeny, v.65, n.1, p.1-8, 2010. Disponível em: <www.jswconline.org/content/65/1/ 1.refs>. Acesso em: 22 jul. 2009. doi: 10.2489/jswc.65.1.1.

YU, L.; OLDFIELD, F. A multivariate mixing model for identifying sediment source from magnetic measurements. Quaternary Research, San Diego, v.32, p.168-181, 1989. Disponível em: <http://www.linkinghub.elsevier.com/retrieve/ pii/0033589489900732>. Acesso em: 03 out. 2006. doi: 10.1016/0033-5894(89)90073-2.

ZAPATA, F. Handbook for the assessment of soil erosion and sedimentation using environmental radionuclides. Dordrecht: Kluwer Academic, 2002. 219p. 